\title{
Introduction to the Cycle of Articles Devoted to Methods and Means of Evaluation and Prediction of Radiation Stability of Submicron Articles of Microelectronics
}

DOI: $10.1134 / \mathrm{S} 1063739709010016$

\section{Simulation and Analysis of Radiation Effects in Microelectronic Devices}

In connection with the expansion of the development and production of submicron VLSI circuits in Russia, the necessity of analyzing their radiation stability to types of radiation environment of varying character involving the effect of separate nuclear particles appears. Therefore, the need arises for further development of the methods and means of evaluation and prediction of radiation stability of modern microelectronics articles of high functional stability performed by submicron rates.

This cycle of articles is a continuation of a cycle of works published in Russian Microelectronics no. 2, 2004; no. 3, 2006; and no. 1, 2008.

In the series of articles called to your attention, the results of practical approbation of laser imitation methods of simulation of dose rate effects in integrated circuits and semiconductor devices are considered. The effect of surface recombination on radiation resistance of bipolar microcircuits is analyzed. A mathematical tool for analysis of the radiation behavior of integrated circuits at the functional-logic level is given and considered.

Special attention is paid to the questions of the use of submicron CMOS VLSI circuits in faulty resistant devices operating under the effect of atmospheric neutrons and extreme thermal modes.

The presented articles reflect modern tendencies in the development of investigations in the field of analysis and monitoring of radiation stability of submicron articles of microelectronics based on wide use of numerical methods of simulation and imitation effects. 